\title{
Effects of exercise training on stroke risk factors, homocysteine concentration, and cognitive function according the $A P O E$ genotype in stroke patients
}

\author{
Joon-Hee Lee', Sang-Min Hong', Yun-A Shin ${ }^{3, *}$ \\ 'Department of Sports Coaching, College of Physical Education, Kyung Hee University, Yongin, Korea \\ 2Department of Physical Education, College of Education, Dongguk University, Seoul, Korea \\ ${ }^{3}$ Department of Prescription \& Rehabilitation of Exercise, College of Physical Exercise, and Department of Kinesiologic Medical Science, Graduate School Dankook \\ University, Cheonan, Korea
}

https://doi.org/10.12965/jer.1836108.054

J Exerc Rehabil 2018;14(2):267-274.

To the editor:

Thanks for giving us the opportunity to contribute to Journal of Exercise Rehabilitation entitled "Effects of exercise training on stroke risk factors, bomocysteine concentration, and cognitive function according the APOE genotype in stroke patients." We found that there is mistake printing regarding the 1st author's name and affiliation. This mistake was not due to editorial office. We attached a corrigendum along with this letter to request for a correction of the 1st author's name and affiliation.

Best Regards,

Yun-A Shin

Corrected the 1 st author's name and affiliation

"Jun-Hee Lee" should be corrected as "Joon-Hee Lee."

"Department of Physical Education, Centre for Sport Medicine, Kyung Hee University, Seoul, Korea" should be corrected as "Department of Sports Coaching, College of Physical Education Kyung Hee University, Yongin, Korea." 